\title{
Assessment of Rayleigh and non-Rayleigh Contributions to the Transmission Losses in fs-Laser Written Er/Yb- Codoped Phosphate Glass Waveguides
}

\author{
Juan A. VALLES ${ }^{* 1}$, Javier SOLIS ${ }^{* 2}$, José A. SANCHEZ-MARTIN ${ }^{* 1}$, Alexandro RUIZ DE LA CRUZ ${ }^{* 2}$, Miguel A. \\ REBOLLEDO*1, and Andrés FERRER $^{* 2}$ \\ ${ }^{* 1}$ Department of Applied Physics-I3A, Universidad de Zaragoza, 50009 Zaragoza, Spain \\ E-mail: juanval@unizar.es \\ ${ }^{* 2}$ Laser Processing Group, Instituto de Optica-CSIC, Serrano 121, 28006 Madrid, Spain
}

\begin{abstract}
A detailed characterization procedure based on the numerical fit of a reduced number of measurements has been applied to two fs-laser written Er/Yb-codoped phosphate glass waveguides. One of them was written in a single scan writing process using higher peak power pulses, and the other one by using lower energy pulses and scanning the laser repetitively over the same region 20 times. The relative weight of non-Rayleigh transmission-loss mechanisms is analyzed for the different processing conditions. As a result of these measurements a quality parameter for fs-laser written active waveguides is proposed.

DOI: $10.2961 /$ jlmn.2010.01.0009
\end{abstract}

Keywords: fs-laser writing, active integrated waveguides, characterization techniques, rare earth ions, optical amplifiers.

\section{Introduction}

The development of fs-laser writing techniques has enabled the production of 3-D waveguiding structures [1]. By doping these structures with rare earth ions, active photonic devices can be fabricated in high-density integrated systems. Among these devices, integrated waveguide lasers stand out due to their strong potential in applications demanding compact, stable and energy efficient devices [2].

The desirable optimization of the performance of active integrated devices requires not only the improvement of the fabrication procedures (i.e the use of high rep. rate fs lasers [3], the use of spatial shaping techniques [4], or the control of aberration effects [5]...) but also a detailed modeling of the propagation of the optical powers (pump, signal and coand counterpropagating amplified spontaneous emission) inside the waveguide and a precise determination of the set of parameters that are included in the model. In particular, the characterization of fs-laser written waveguides involves additional difficulties that require the development of specific techniques. Since they are written inside the material, they are not transversally accessible, which forces using characterization techniques exclusively based on the measurement of in- and outcoupled optical powers [6]. In addition these waveguides show transmission losses with a wavelength dependence that has been shown in some cases to deviate from the $\lambda^{-4}$ Rayleigh scattering scaling [7].

Usually, a single transversal fs-laser writing scan process is carried out in order to generate a waveguide [8]. However, in order to generate the necessary refractive index variation in a single scan, writing requires high energy pulses that cause unwanted nonlinear effects such as selffocusing, beam filamentation or nonlinear absorption in the prefocal region [9] . In order to avoid these problems writing with low energy pulses has been proposed as an efficient alternative [10] in order to deposit most of the energy in the focal volume region. As a consequence a very small refractive index increase is reached, and then the waveguide has to be rescanned in order to achieve proper guiding conditions. This way, the lower stress generated amongst the pre and post focal volume regions (always along the writing direction) should also contribute to a significant decrease of non-Rayleigh scattering loss (stressed regions with sizes in the $\mu \mathrm{m}$ range).

It this context it must be considered that the laser writing process may induce, depending on the material and the processing conditions, the formation of sidewalls roughness and scattering centers including nano-gratings with spacing's of the order of $\lambda / 2$ [11].When these structures present a sub-wavelength size, they can contribute to Rayleigh type losses. Additionally, the formation/redistribution of absorption centers may alter the wavelength dependence of losses, leading to a nonRayleigh type contribution

In this paper we apply a characterization procedure, that we have previously developed [12], in order to analyze the performance of two waveguides written respectively with laser pulses above and below the nonlinear beam filamentation threshold. For the former, the waveguide was written in a single-scan step whereas for the later a multiscan procedure was used. The best-fit parameters for both waveguides are compared and the dependence on the process conditions of the relative weight of Rayleigh and nonRayleigh transmission-loss mechanisms is analyzed. Finally, a quality parameter that readily describes the relative influence of non-Rayleigh scattering losses is proposed.

\section{Experimental}

2.1 The laser written waveguides. 
Both waveguides were written in a commercial $\mathrm{Er}: \mathrm{Yb}$ co-doped phosphate glass (MM2, Kigre Inc.) and processed by using a commercial amplified fs laser system (100 fs) working at a repetition rate of $1 \mathrm{kHz}$. The beam, circularly polarized to minimize the potential formation of nanogratings [11], was shaped by placing a slit (width $=350 \mu \mathrm{m}$ ) in order to get a disk-shaped focal volume [13]. The focusing optics was a 10x microscope objective $(\mathrm{NA}=0.26)$, the scanning speed was $100 \mu \mathrm{m} / \mathrm{s}$ and the processing depth was set to $625 \mu \mathrm{m}$. The pulse duration was stretched to $266 \mathrm{fs}$ in order to optimize the energy deposition in the focal volume region in the waveguides written with low energy pulses [10].

Waveguide \#1 was 9.5-mm long and the glass was codoped with $\sim 2 \% \mathrm{Er}$ and $\sim 2.1 \% \mathrm{Yb}$ (wt.\%). The pulse energy was set to $8 \mu \mathrm{J}$, above the non-linear beam filamentation threshold [10]. Waveguide \#2 was $16.05-\mathrm{mm}$ long and was written in a similar phosphate glass codoped with

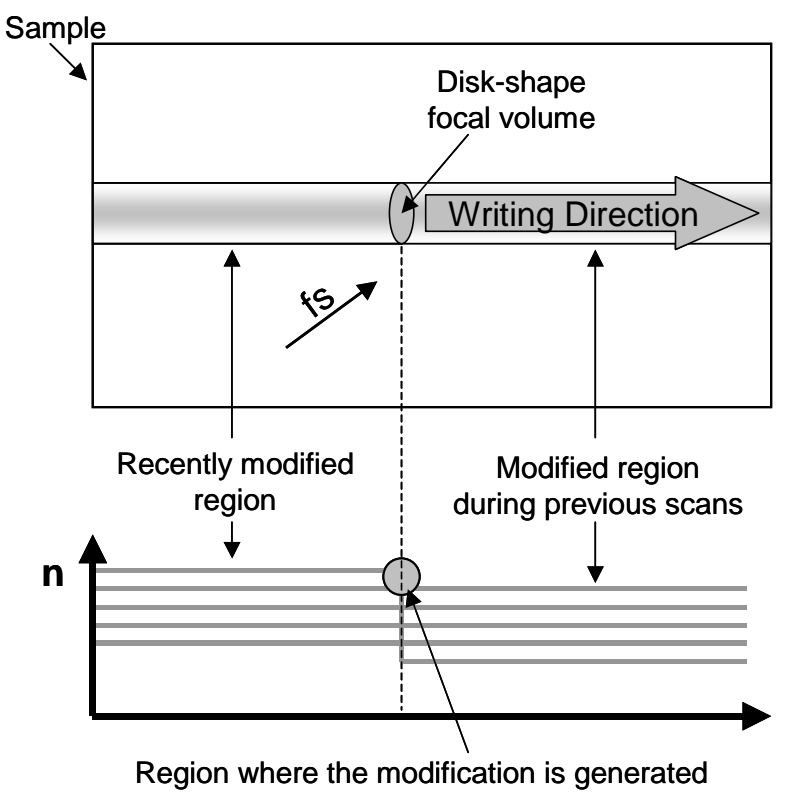

(b)

Fig. 1. Schematics of the fabrication process of fs-laser written waveguides for (a) single writing scan and (b) multiscan writing.

$\sim 2.6 \% \mathrm{Er}$ and $2.9 \% \mathrm{Yb}(\mathrm{wt} . \%)$ instead. $4-\mu \mathrm{J}$ energy pulses were used to suppress beam filamentation effects and 20 scans were carried out. In Fig. 1 the schematics of both writing procedures are shown. Further details regarding the experimental set up used for waveguide writing can be found in [8]. Whereas both waveguides showed single mode propagation at the signal wavelength, the pump power propagated in a multimode regime.

\subsection{The characterization setup and measurements.}

For the characterization of each waveguide an optical amplifier was built using the waveguide as the active medium. The pump laser was a 980 -nm semiconductor laser diode and two 980/1550-nm Wavelength Division Multiplexers (WDMs) allowed incoupling the pump and the copropagating signal and outcoupling the amplified signal, the remaining pump and the co- and counterpropagating amplified spontaneous emission (ASE ${ }^{ \pm}$. Pump power attenuation, $\mathrm{ASE}^{ \pm}$power spectra and small signal gain measurements were carried out for a large pump power range. The maximum available pump power at the waveguide input end was measured to be $245 \mathrm{~mW}$ (for a $900 \mathrm{~mA}$ laserdiode drive current). The outcoupled optical powers were measured for nine equally-spaced values of the laser-diode drive-current. Due to the multimode regime for the pump propagation inside the waveguide, the outcoupled pump power was measured with a $50 \mu \mathrm{m}$-diameter fibre. The attenuation of the pump in the waveguide was obtained from the measurement of the in- and output pump powers. The $\mathrm{ASE}^{ \pm}$power spectra were registered from 1500 to 1600 $\mathrm{nm}$ with an Optical Spectrum Analyser (OSA). For the small signal gain measurements a tunable laser was used as the signal source, with an output power of $3.8 \mathrm{nW}$ at 1534 $\mathrm{nm}$, which was the peak wavelength of the $\mathrm{ASE}^{ \pm}$spectra. Finally, the signal power attenuation in absence of pump was also measured.

\section{Results and discussion.}

\subsection{Considerations regarding the wavelength depend- ence of losses.}

In [12] a numerical fit procedure for pigtailed packaged waveguides (not transversally accessible) was adapted for active fs-laser written waveguides. Whereas most waveguide fabrication techniques generate smooth refractive index profile variations leading to transmission losses 
due to Rayleigh scattering (scaling as $\lambda^{-4}$ ), fs-laser writing may produce inhomogeneities, sidewall roughness and other loss mechanisms, which become relevant. As a consequence, for this type of waveguides the transmission loss ratio $\alpha(980 \mathrm{~nm}) / \alpha(1534 \mathrm{~nm})$ is beforehand unknown. In order to determine this value, the dependence on the input pump power of the ratio between the counter- and copropagation ASE at the spectra peak wavelength was also fitted. This ratio is strongly influenced by the transmission loss ratio and by any potential unbalance between insertion loss values at both waveguide ends. Moreover, as in the case of pump power attenuation and small signal gain (the other measurements that are used for the numeri- cal fit process), the use of the dependence of $A^{-} E^{-}(1534$ $\mathrm{nm}) / \mathrm{ASE}^{+}(1534 \mathrm{~nm})$ on the input pump power avoids inaccuracies associated to absolute optical power measurements.

\subsection{Comparison of waveguides written above and be- low the non-linear filamentation threshold}

The three-step numerical fitting process was then applied to both waveguides. In table I the best-fit parameters are summarized. An excellent agreement is observed in the values of the $\mathrm{Yb}^{3+}$-ion absorption cross section and in the coefficients of both energy-transfer mechanisms, $\mathrm{Yb}^{3+} \Rightarrow$ $\mathrm{Er}^{3+}$ energy transfer and $\mathrm{Er}^{3+}$ upconversion [6]. This agreement confirms the robustness of the numerical fitting

Table I. Values of the best-fit parameters for both waveguides \#1 and \#2

\begin{tabular}{ccc}
\hline Best-fit parameter & Waveguide \#1 & Waveguide \#2 \\
\hline Transmision loss $(980 \mathrm{~nm})$ & $6.3 \mathrm{~dB} / \mathrm{cm}$ & $3.6 \mathrm{~dB} / \mathrm{cm}$ \\
Transmision loss $(1534 \mathrm{~nm})$ & $5.3 \mathrm{~dB} / \mathrm{cm}$ & $0.6 \mathrm{~dB} / \mathrm{cm}$ \\
Input end coupling loss $(980 \mathrm{~nm})$ & $2.6 \mathrm{~dB}$ & $1.2 \mathrm{~dB} / \mathrm{cm}$ \\
Input end coupling loss $(1534 \mathrm{~nm})$ & $2.1 \mathrm{~dB}$ & $0.3 \mathrm{~dB} / \mathrm{cm}$ \\
Output end coupling loss $(980 \mathrm{~nm})$ & $1.4 \mathrm{~dB}$ & $1.2 \mathrm{~dB} / \mathrm{cm}$ \\
Output end coupling loss $(1534 \mathrm{~nm})$ & $0.9 \mathrm{~dB}$ & $0.3 \mathrm{~dB} / \mathrm{cm}$ \\
$\mathrm{Yb}^{3+}$ absorption cross section $(980 \mathrm{~nm})$ & $5.3 \times 10^{-25} \mathrm{~m}^{2}$ & $5.3 \times 10^{-25} \mathrm{~m}^{2}$ \\
$\mathrm{Yb}^{3+} \Rightarrow$ Er$^{3+}$ energy transfer coefficient & $1.8 \times 10^{-23} \mathrm{~m}^{3} / \mathrm{s}$ & $1.8 \times 10^{-23} \mathrm{~m}^{3} / \mathrm{s}$ \\
$\mathrm{Er}^{3+}$ upconversion coefficient & $5.2 \times 10^{-24} \mathrm{~m}^{3} / \mathrm{s}$ & $5.6 \times 10^{-24} \mathrm{~m}^{3} / \mathrm{s}$ \\
Incoupled pump additional attenuation & $7.5 \mathrm{~dB}$ & $8.5 \mathrm{~dB}$ \\
Transmission loss ratio & 1.2 & 6.0 \\
Quality parameter $\left(\lambda^{-n}\right)$ & 0.4 & 4 \\
\hline
\end{tabular}

process. If the transmission losses are compared, they are significantly lower at the pump wavelength in waveguide $\# 2$, furthermore, at the signal wavelength this difference is larger. Accordingly, the transmission loss ratio changes from 1.2 for waveguide \#1 to 6.0 for waveguide $\# 2$, which is the expected value for this ratio in case of a $\lambda^{-4}$ dependence. This difference confirms the predominance of other loss mechanisms over Rayleigh scattering for the single scan written waveguide, and how these mechanisms have a

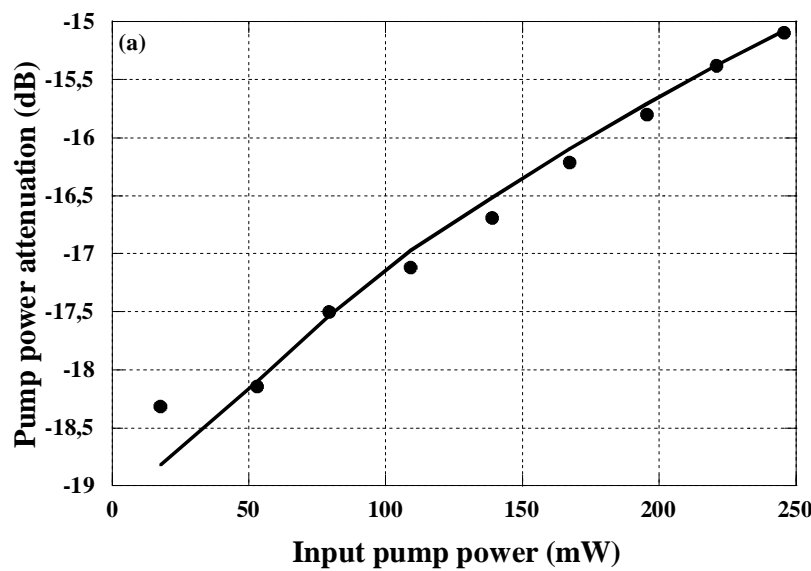

larger effect for longer wavelengths. Moreover, if a power dependence on wavelength, $\lambda^{-n}$, is assumed for transmission losses on wavelength, the exponent $n$ acts as a kind of waveguide quality parameter. The closer this parameter value is to 4 , the less significant are the non-Rayleigh scattering loss mechanisms. In addition, insertion losses are clearly lower in waveguide \#2 for both pump and signal wavelengths.

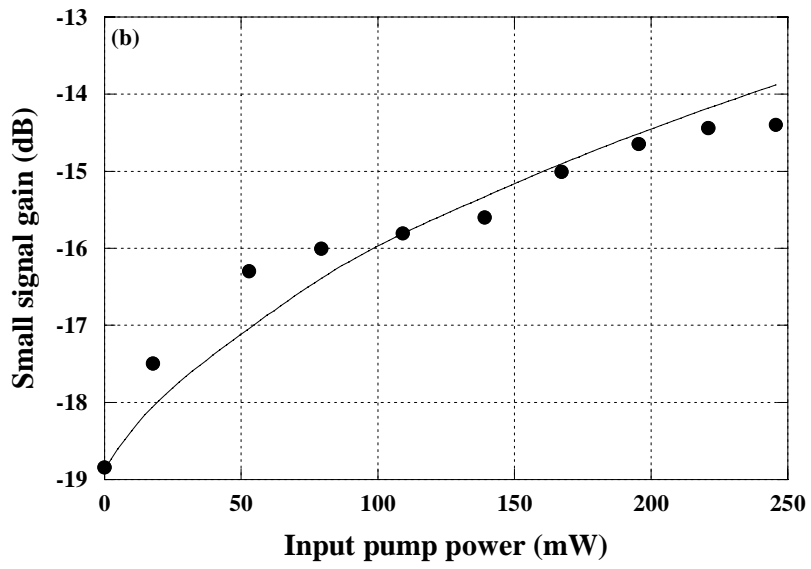

Fig. 2. Measured (a) pump power attenuation and (b) small signal gain inside the waveguide $(\bullet)$ and numerically calculated values for the best-fit parameters in table I (full line), as a function of the input pump power. 
Finally, the additional pump attenuation values due its multimode behaviour have been experimentally confirmed by comparing them to the decrease in the output pump power values if an outcoupling single mode fiber is used instead.

The measured and the numerically calculated values for the best-fit parameters of the pump power attenuation and of the small signal gain as a function of the input pump power are shown in fig. 2 for waveguide \#2. Both dependences present a good agreement.

\section{Conclusions}

The complete characterization of two fs-laser written waveguides (one in a single writing scan process and the other in a multiscan writing procedure) allows the analysis of the dependence of transmission loss ratio on the processing conditions and the determination of the relative weight of non-Rayleigh loss mechanisms. If, in any case, a $\lambda^{-n}$ power dependence is assumed for transmission losses as a function of the wavelength, the exponent $n$ acts as a kind of waveguide quality parameter for fs-written waveguides: the closer $n$ is to 4 that this parameter is, the less the dependence on non-Rayleigh scattering loss mechanisms.

\section{Acknowledgments}

This work was supported by the Comisión Interministerial de Ciencia y Tecnología, projects FIS2006-03639 and TEC2008-01183 and by the Diputación General de Aragón. Andrés Ferrer acknowledges a grant under Projects TEC 2006-04538. Alexandro Ruíz de la Cruz acknowledges a I3P-CSIC postdoctoral contract co-founded by the European Social Fund.

\section{References}

[1] K.M. Davis, K. Miura, N. Sugimoto, K. Hirao: Opt. Lett. 21 (1996) 1729-31.

[2] S. Tacheo, G. della Valle, R. Osellame, G. Cerullo, N. Chiodo, P. Laporta, O. Svelto, A. Killi, U. Morgner, M. Lederer, D. Kopf: Opt. Lett. 29, (2004) 2626-8.

[3] C. B. Schaffer, J. F. García, and E. Mazur: Appl. Phys. A 76, (2003) 351-4.

[4] V. Diez-Blanco, J. Siegel, A. Ferrer, A. R. D. L. Cruz, and J. Solis: Appl. Phys. Lett. 91, (2007) 051104.

[5] C. Hnatovsky, R. S. Taylor, E. Simova, V. R. Bhardwaj, D. M. Rayner, P. B. Corkum: J. Appl. Phys. 98, 013517 (2005)

[6] J. A. Vallés, M. A. Rebolledo and J. Cortés: IEEE J. Quantum Electron. 42, (2006) 152-9.

[7] L. Tong, R. R. Gattass, I. Maxwell, J. B. Ashcom, E. Mazur: Opt. Comm. 259, (2006) 626-39.

[8] A. Ferrer, V. Díez-Blanco, A. Ruiz, J. Siegel and J. Solís: Appl. Surf. Sci. 254, (2007) 1121-5

[9] D. M. Rayner, A. Naumov and P. B. Corkum: Opt. Express 13, (2005) 3208-3217.

[10] W. Gawelda, D. Puerto, J. Siegel, A. Ferrer, A. Ruiz de la Cruz, H. Fernández and J. Solis: Appl. Phys. Lett., 93, (2008) 121109.
[11] Y. Shimotsuma, P. G. Kazansky, J.Qiu, K Hirao: Phys.Rev.Lett. 91, 247405-1-4, (2003)

[12] J. A. Vallés, J. Solís, J. A. Sánchez-Martín, A. Ferrer, M. A. Rebolledo, A. Ruiz de la Cruz: submitted to Journal of Quantum Electronics.

[13] M. Ams, G. D. Marshall, D. J. Spence, and M. J. Withford: Opt. Express 13, (2005) 5676-81.

(Received: July 11, 2009, Accepted: January 8, 2010) 